\title{
Lessons and Challenges to be learned from different countries policy implication on COVID 19 recovery cases- A cross-sectional descriptive study
}

NAIYA PATEL ( $\square$ naiya.patel@louisville.edu )

\section{Research}

Keywords: COVID-19, Health Policy, Social distancing

Posted Date: April 9th, 2020

DOI: https://doi.org/10.21203/rs.3.rs-21447/v1

License: (c) (1) This work is licensed under a Creative Commons Attribution 4.0 International License.

Read Full License 


\section{Abstract}

Background- The need to quantify the non-pharmaceutical measures in policy decision making is essential in current uncertain times of pandemic. The purpose of the current study is to quantify the relationship between Social Distancing measures and the Total number of tests performed with the Total number of recovered cases across 23 countries around the world, currently struck by COVID-19 pandemic.

Methods- The cross-sectional descriptive study utilized STATA 16. for Poisson Model analysis using data collected across 23 countries. The statistical databases Statista, WHO situation reports, CDC website, respective country health ministry websites, and World Bank data was utilized to collected the lacking data details regarding COVID-19. The WHO regions/23 countries included in analysis are Republic of Korea, Japan, Australia, Malaysia, Philippines, Thailand, India, United States of America, Canada, Italy,Germany,United Kingdom,France,Austria,Croatia,Israel,Russian Federation,Spain,Belgium,Finland,Sweden,Switzerland,Iran (Islamic Republic of). The variables included in analysis are The factorial analysis of categorical data is included to quantify the levels of social distancing measures and its effect on the total number of recovered cases until April $2^{\text {nd, }} 2020$.

Results- There exists a positive relationship between the improved number of recovered infected cases, and Social distancing measures of lockdown, the total number of tests performed depending on the stage at which it is completed. The availability of total medical doctors in each country affects the number of recovered cases in that particular country.

Conclusion- Future studies might use it as a foundation for evaluation modeling in public health for policy decision making.

\section{Background}

The uncertainty about COVID-19 in terms of treatment decision making, confinement, and bringing down the trajectory, has alarmed health authorities around the world. ${ }^{1}$ The pandemic has caused crises in certain countries, now identified as widespread transmission countries by $\mathrm{CDC} .^{2}$ The daily uncertainty about the trends and inability to predict the course of COVID 19 has caused disruption worldwide from quarantine to non-pharmaceutical measures. ${ }^{2}$ The COVID-19 spread has been tracked down to China and later a spillover to several parts of the globe currently. ${ }^{1}$ Fig. $1^{3}$ depicts the number of COVID-19 cases worldwide by country and place of exposure as of February 26, 2020, an initial outbreak of the about to be pandemic. ${ }^{3}$ The spillover has already occurred, and currently, we have already surpassed the preventive stage worldwide. The question now is when will the uncertainty become certain? Without much accurate data availability, it is difficult to understand the trends and potential solutions of the pandemic.

\section{Purpose}


The current study aims to determine any linear correlational association with the total recovered cases to quantify if the current public health measures are useful in real-time. With the lack of accurate, detailed data and an evaluation model, it becomes challenging for the health authorities and policymakers to come to an outlook. The data collected from multiple reliable health ministry websites of 23 countries, WHO situation reports, CDC website, and secure database Statista, is one of the only cross-sectional data to the author's knowledge. The WHO regions/23 countries included in analysis are Republic of Korea, Japan, Australia, Malaysia, Philippines, Thailand, India, Italy,Germany,United Kingdom,France,Austria,Croatia,Israel,Russian Federation, Spain, Belgium, Finland, Sweden, Switzerland, Iran (Islamic Republic of), United States of America and Canada. The study aims to employ a Poisson Regression Model for correlations analysis to evaluate non-pharmaceutical measures like Social distancing laws, total tests performed, number of days it too to double the number of new infected cases in each region, and its relationship to total recovered cases around the world as of April $2^{\text {nd, }} 2020$. Research studies so far are more focused on the molecular aspect. However, while the treatment is tested, the constant evaluation of the effectiveness of non-pharmaceutical measures is critically essential to contain the pandemic and potential associated loss.

\section{Methods}

The cross-sectional study captures 23 countries. It includes the Total number of new cases, Total tests Performed, Healthcare Workforce, Total deaths, Active cases, New Deaths, Total recovery, Social distancing, and traveling measures, Total number of Hospital beds, Total Serious Critical cases as of April $2^{\text {nd, }}$ 2020. The Statistical software used for analysis is STATA.16, and the model used to conclude is the Poisson Regression Model. The continuous dependent variable is Total Recovered cases across 23 countries and the independent variable with covariates are Total number of tests performed, Total number of Hospital beds of each country, Total Country Population, Days it took in each country to

double the number of infected cases. ${ }^{4-6}$ The categorical variable of Social distancing measures- School, Mass gathering, Sports Events, Restaurants, National, and International Travelling- was converted into factor variables to fit in the model analysis. The Values from 1 to 4 were provided in each category beginning from 1 to be no restrictions of governing authorities in the country until March 30th to 4, which meant complete lockdown and self-quarantine laws. The hypothesis of the study is as follows:

$\mathrm{H}_{0}=$ The total number of recovered cases doesn't vary depending on the total number of tests performed, Total number of Doctors and Nurses, Total Country Population, Days it took in each country to double the number of infected cases, and Social distancing measures.

$\mathrm{H}_{1}=$ The total number of recovered cases does vary depending on the total number of tests performed, the Total number of Doctors and Nurses, Total Country Population, Days it took in each country to double the number of infected cases and Social distancing measures.

\section{Results}


The chi2 p-value indicates that the model is statistically significant for the independent variables' coefficients. The descriptive statistics of the Outcome/ Dependent variable was highly skewed, and after careful descriptive analysis, Poisson Model is utilized.

The results Table 1 depicts the Poisson Model for regression techniques. The Days to double the infection case and Total Population seems to have a negative relationship with the Total number of recovered cases across countries. With a slight decrease in the total number of days, The chances of Total recovery improves by $29 \%$. The Total country population seems to have a negative effect on the Total number of recovered cases, and every increase by 5.3 in Population total decreases the chances of Total recovery by that unit. Increased levels of Social distancing measures in Schools and Mass Gatherings improve the chances of Total recovered cases improvement by 4.17 and 3.75, respectively. An increase in the Total number of Medical doctor's availability improves the chances of total recovery by 8.25 units of the coefficient. The p-values $(0.00)$ of all the covariates and independent variables are statistically significant, with the alpha value set at 0.05 . Hence we have enough evidence to reject the null hypothesis. 
Poisson Regression Model results

\section{Poisson regression Number of obs $=23$}

LR chi2(14) = 163317.33

Prob $>$ chi2 $=0.0000$

Log likelihood $=-32466.374$ Pseudo R2 $=0.7155$

TOTrec

Coef.

Std. Err.

$z \quad P$

P [95\% Conf. Interval]

$\mathbf{Z}$

Days2

$-0.294122 \quad 0.0028653$

$-102.65$

$-0.2997379$

$-0.2885061$

Nursenum

$-5.32 \mathrm{E}-07$

8.99E-09

$-59.23$

$-5.50 \mathrm{E}-07$

$-5.15 \mathrm{E}-07$

Docnum

8.25E-07

2.75E-08

30.03

7.71E-07

8.78E-07

TOTtest

5.43E-06

3.07E-08

176.9

5.37E-06

5.49E-06

Poptot

$-2.11 \mathrm{E}-09$

3.39E-11

$-62.32$

$-2.18 \mathrm{E}-09$

$-2.05 \mathrm{E}-09$

schools

\begin{tabular}{lllllll}
3 & -0.736864 & 0.0532231 & -13.84 & 0 & -0.8411794 & -0.6325487 \\
\hline 4 & 4.170358 & 0.0711063 & 58.65 & 0 & 4.030993 & 4.309724
\end{tabular}

restaurants

\begin{tabular}{lllllll}
3 & -4.534152 & 0.0921385 & -49.21 & 0 & -4.71474 & -4.353564 \\
\hline 4 & -4.972892 & 0.093186 & -53.37 & 0 & -5.155533 & -4.79025 \\
\hline 4.sportsevents & 0 & $\begin{array}{l}\text { (omitted because } \\
\text { of collinearity) }\end{array}$ & & & & \\
& & & & &
\end{tabular}

massgathering

\begin{tabular}{|lllllll|}
\hline 3 & 2.955872 & 0.0683015 & 43.28 & 0 & 2.822003 & 3.08974 \\
\hline 4 & 3.748888 & 0.0661711 & 56.65 & 0 & 3.619195 & 3.878581 \\
\hline 4.internationaltravel & -1.224956 & 0.0129123 & -94.87 & 0 & -1.250263 & -1.199648 \\
\hline nationaltravel & & & & & & \\
\hline 2 & -4.119077 & 0.0673854 & -61.13 & 0 & -4.25115 & -3.987004 \\
\hline 3 & -4.048821 & 0.0629116 & -64.36 & 0 & -4.172125 & -3.925516 \\
\hline Ccons & -3.008197 & 0.0613292 & -49.05 & 0 & -3.1284 & -2.887994 \\
\hline
\end{tabular}




\section{Discussion}

More the tests performed and time or stage of virulence at which the test is performed has a positive effect on total recovery, as seen in the Poisson model. Similarly, the longer the number of days it takes to double the cases, the higher the chances of improved recovery cases in a specific country. It also means that the current social distancing measures would keep the number of people getting infection away and hence would take longer to infect more people. It also quantifies how the need for total medical doctor's play a vital role in improving total recovery cases. The Social distancing measures are quantified as well, which implicates that stricter rules of lock downplay a crucial decisive role in attaining higher recovery cases. Higher the Population or crowded the area, the lesser the chances of increased recovered cases.

The negative association of higher levels of social measures of traveling seems to quantify a negative association with total cases of recovery. It might indicate the several individual-level factors from genetics to chronic disease and other sociodemographic factors, which currently isn't available for analysis. The travel might be due to medical needs or social isolation of infected individuals. The negative association of total lockdown of restaurants might partly explain the necessity of food by individuals who depend on restaurants for lunch, breakfast, and dinner. Finally, a negative relationship of the Total number of nurses with total recovered cases might fail to explain details of the relationship, given the constraints of current data dearth on COVID-19.

\section{Policy Implications}

An emerging need for better data to apply an appropriate model for interaction at each level is required to predict the certainty of the COVID-19 pandemic. The necessity of accurate data to evaluate the public health and non-pharmaceutical measures is utterly essential to understand and redistribute the available resources in confining this pandemic and save several lives along with the potentially drowning economy. For health policymakers and government authorities, the shortage of such a prediction and evaluation model might delay the response for concluding and appropriate public health measures. As statistical models are vital to quantify and scale-up the redistribution of available resources strategically efficiently, in confining the infections. The need to understand what the hidden consequences of total quarantine are, detailed data in this critical scenario is challenging to get. The policy implications with apparent and hidden consequences and challenges need a statistical model to better scale up the results smoothly.

\section{Conclusion}

The current research has limitations in terms of the inability to explain certain variables relationship with outcome variable due to lack of substantial detailed data requirement and unavailability at the time. Future studies might use it as a foundation for evaluation modeling in public health for policy decision making.

\section{Declarations}


Ethics approval and consent to participate- No human research participants or animals were involved

Consent for publication - Not applicable

Availability of data and material- The datasets during and/or analysed during the current study available from the corresponding author on reasonable request.

Competing interests- The authors declare that they have no competing interests

Funding- None

Authors' contributions- The author has made substantial contribution

Acknowledgements- None

Author's information- The author works for the National Science Foundation Funded Research Center

\section{References}

1. Lin C, Braund WE, Auerbach J, Chou JH, Teng JH, Tu P, Mullen J. Policy Decisions and Use of Information Technology to Fight 2019 Novel Coronavirus Disease, Taiwan.

2. Gostin LO, Hodge JG. US Emergency Legal Responses to Novel Coronavirus: Balancing Public Health and Civil Liberties. Jama. 2020 Feb 13.

3. WHO COVID-19 situation report 37 [Internet]. Www-statista-com.echo.louisville.edu. 2020 [cited 5 April 2020]. Available from: https://www-statista-com.echo.louisville.edu/statistics/1093252/novelcoronavirus-2019ncov-cases-worldwide-by-country-and-transmission-location/

4. GHO By category Health workforce [Internet]. Apps.who.int. 2020 [cited 5 April 2020]. Available from: https://apps.who.int/gho/data/node.main.HWF

5. Coronavirus Testing - Source Data [Internet]. Our World in Data. 2020 [cited 5 April 2020]. Available from: https://ourworldindata.org/coronavirus-testing-source-data

6. World Development Indicators DataBank [Internet]. Databank.worldbank.org. 2020 [cited 5 April 2020]. Available from: https://databank.worldbank.org/indicator/SP.POP.TOTL/1ff4a498/PopularIndicators

\section{Figures}




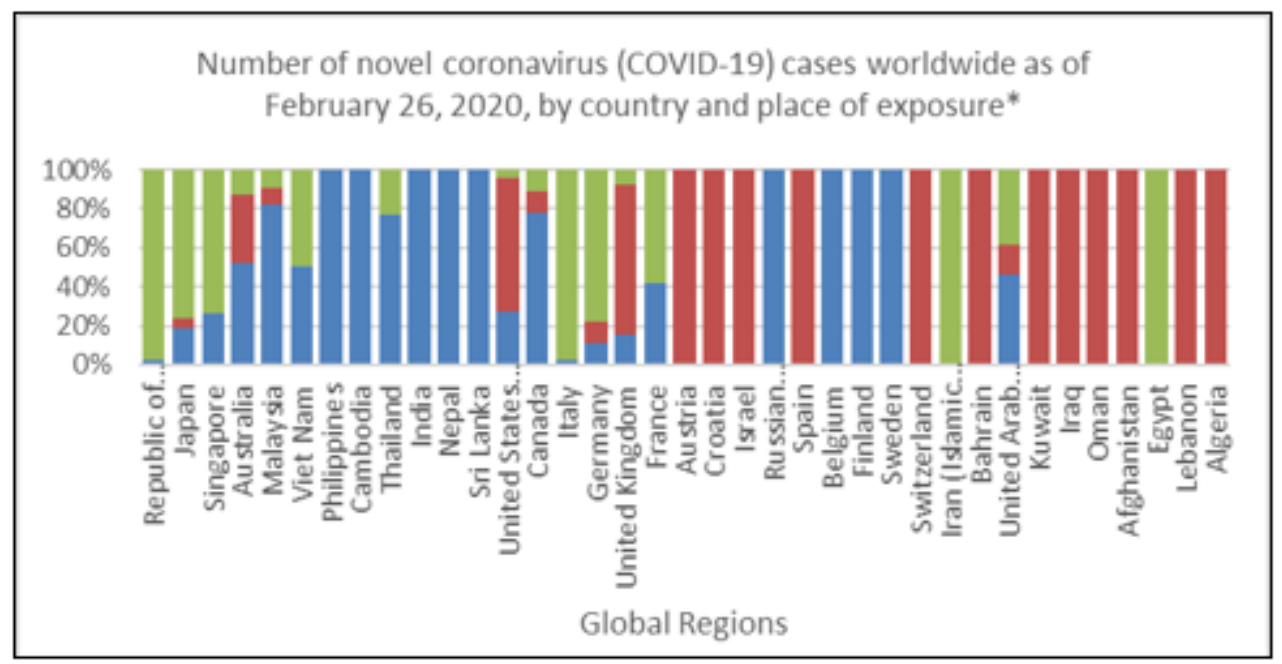

Legends- $\quad$ China $\quad$ Outside reporting country and outside China $\mathbf{m}$ In reporting country

Figure 1

Number of COVID-19 cases worldwide by country and place of exposure 